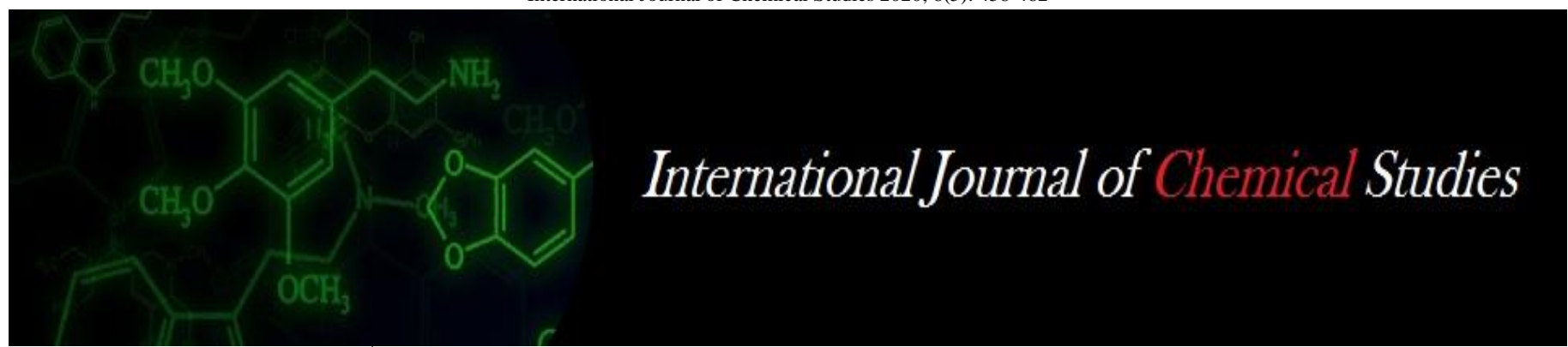

P-ISSN: 2349-8528

E-ISSN: 2321-4902

www.chemijournal.com

IJCS 2020; 8(5): 458-462

(C) 2020 IJCS

Received: 01-07-2020

Accepted: 03-08-2020

\section{Sharvan Kumar}

Ph.D. Scholar, Department of

Vegetable Science, Acharya

Narendra Deva University of

Agriculture \& Technology,

Kumarganj, Ayodhya,

Uttar Pradesh, India

\section{VB Singh}

Associate Professor, Department of Vegetable Science, Acharya

Narendra Deva University of

Agriculture \& Technology,

Kumarganj, Ayodhya,

Uttar Pradesh, India

\section{Manjeet Kumar}

Ph.D. Scholar, Department of

Vegetable Science, Acharya

Narendra Deva University of

Agriculture \& Technology,

Kumarganj, Ayodhya,

Uttar Pradesh, India

\section{Pankaj Kumar Verma}

Ph.D. Scholar, Department of

Vegetable Science, Acharya

Narendra Deva University of

Agriculture \& Technology,

Kumarganj, Ayodhya,

Uttar Pradesh, India

\section{Yogesh Kumar}

Associate Professor, Department of Vegetable Science, Acharya

Narendra Deva University of

Agriculture \& Technology,

Kumarganj, Ayodhya,

Uttar Pradesh, India

\section{Study on inter traits relationship among the parents and hybrids for seed yield and its contributing traits in fenugreek (Trigonella foenum-graecum $\mathrm{L}$.)}

\author{
Sharvan Kumar, VB Singh, Manjeet Kumar, Pankaj Kumar Verma and \\ Yogesh Kumar
}

DOI: https://doi.org/10.22271/chemi.2020.v8.i5g.10339

\begin{abstract}
The present investigation was carried out at Main Experiment Station, Department of Vegetable Science, Acharya Narendra Deva University of Agriculture \& Technology, Kumarganj, Ayodhya (U.P.). The characters studied were Days to 50\% flowering, Days to maturity, Plant height (cm), Number of branches per plantv, Pod length, Number of pod per plant, Number of seed per pod, Test weight (g), Biological yield $(\mathrm{g})$, Harvest index $(\%)$ and Harvest index $(\%)$. At phenotypic level seed yield per plant exhibited positively significant correlation with harvest index (0.825), Number of pods per plant (0.921), days to maturity (0.374), Number of branches per plant $(0.281)$, plant height $(0.338)$ and pod length $(0.287)$. Harvest index exhibited positively significant correlation with test weight (0.483), number of pod per plant (0.750), plant height (0.412) and days to maturity (0.339). Harvest index was also showed negatively significant correlation with biological yield per plant (-0.618). Biological yield per plant exhibited positively significant correlation with number of branches per plant (0.403), number of seed per pod $(0.341)$ and showed negatively significant correlation with test weight $(-0.501)$ and plant height ($0.271)$. Test weight showed negatively significant correlation with days to $50 \%$ flowering $(0.629)$ and days to maturity (0.406). Number of seeds per pod (0.698). Number of pod per plant was exhibited positively significant correlation with number of branches per plant (0.295), plant height (0.289) and days to maturity (0.304). exhibited positively significant correlation with days to $50 \%$ flowering $(0.560)$. At the genotypic level in general the correlation coefficient were similar in nature but higher in magnitude to that of observed value at phenotypic level. Here also seed yield per plant showed highly significant and positive correlation with harvest index, days to maturity, test weight, plant height, pods length and number of branches per plants. Other combinations of different pair of characters showed association as at phenotypic level during $E_{1}, E_{2}$ and pooled. However as seed yield per plant showed highly significant and negative correlation with biological yield.
\end{abstract}

Keywords: Fenugreek, correlation, yield

\section{Introduction}

Fenugreek (Trigonella foenum-graecum L.) $2 \mathrm{n}=16$, locally known as 'Methi' belongs to the family-Leguminoaceae and sub-family-Papilionaceae, along with it's another cultivated species Trigonella corniculata L. commonly known as Kasuri or Champa methi differing as growth habit, pod seed size and yield potential as serve and multipurpose crop. Fenugreek is grown during Rabi or winter season as a leafy vegetable, seed or leaf spices for human consumption (Som and Maity 1986, Pandey 1993) ${ }^{[13,7]}$ fodder for the animal (Jatasra and Lodhi, 1980) and green manure to enrich the soil fertility through nitrogen fixation, i.e above $283 \mathrm{~kg} \mathrm{~N} / \mathrm{ha}$ (Gill \& Singh 1988) ${ }^{[5]}$. India has always be known as "Home of Spices" where 63 kinds of spices are grown (Pruthi, 1998). According to Spices Board, Culicut, Kerla, mainly 52 spices are grown in India.

It is widely used as spice and condiment to add flavour in various foods (Dwivedi et al., 2006). Fenugreek is cultivated as leafy vegetable, condiment and medicine. Fenugreek which forms the actual spice is rich source of vitamin A, C and $B_{2}$ (Aykroyd, 1963) ${ }^{[3]}$, protein (Rao and Sharma 1987) ${ }^{[11]}$, diosgenin which is extracted from the seeds is used in synthesis of sex hormones. The wild form of fenugreek is found growing in North Western India, Argentina,
Corresponding Author: Sharvan Kumar

Ph.D. Scholar, Department of Vegetable Science, Acharya Narendra Deva University of Agriculture \& Technology,

Kumarganj, Ayodhya,

Uttar Pradesh, India 
Egypt, Southern France, Morocco, Spain, Turkey, China, Pakistan and Lebanon are the leading countries for fenugreek production. Fenugreek is self-pollinated, but there are opportunities for natural out crossing, Allard (1960) ${ }^{[2]}$.

The great extent of natural variation present in various characters among the genotypes suggests good scope of improvement in economic traits. Large variability ensures better chance of producing new forms. Variability parameters like genotypic and phenotypic coefficient of variability, heritability and genetic advance, besides degree of association between the various characters and direct effect of yield contributing characters on total seed yield, is of paramount significance in formulating an appropriate breeding strategy aimed at exploiting the inherent variability of the original population.

Phenotypic variability changes under different environmental conditions while genetic variability remains unchanged and more useful to a plant breeder for exploitation in selection or hybridization. Yield is very complex characteristics controlled by several yield contributing components and it is highly influenced by environmental factors, consequently estimates of heritability and genetic advance are useful for selection. Estimation of correlation coefficient among the yield contributing characters is necessary to understand the direction of selection and maximize seed yield in the shortest period.

\section{Materials and Methods}

The present investigation entitled "Study on inter traits relationship among the parents and hybrids for seed yield and its contributing traits in fenugreek (Trigonella foenumgraecum L.)" was carried out at Main Experiment Station (Vegetable Research Farm), Narendra Nagar (Kumarganj), Ayodhya (U.P.) India, during Rabi season of 2018-19. Geographically the experimental site falls under humid subtropical climate and is located at $26.47^{\circ} \mathrm{N}$ latitude and $82.12^{\circ} \mathrm{E}$ longitude at an elevation of altitude of 113 meter above the mean sea level. Geographically it falls in north east gangatic alluvial plains of eastern U.P. Ayodhya region. The experimental field had sandy loam soil, low in organic carbon, nitrogen, medium in phosphorous, potash and slightly alkaline $(\mathrm{pH} \mathrm{8.0)}$ in nature. The mechanical mixture of soil was $60.9 \%$ sand, $27.8 \%$ silt and $11.3 \%$ clay. The climate of district Ayodhya is semi-arid with hot summer and cold winter. Maximum rains in this area are received from July to the end of September. The winter months are usually cool and dry but occasional light showers are also not uncommon whereas, summer months are extreme hot and dry. The data was recorded at meteorological observatory of Acharya Narendra Deva University of Agriculture and Technology, Narendra Nagar (Kumarganj), Ayodhya (U.P.) The experiment was conducted in a Randomized Block Design (R.B.D.) with three replications to assess the performance of $45 \mathrm{~F}_{1}$ hybrids and their 10 parents and one commercial check. The crop was planted in row length spaced $30 \mathrm{~cm}$. apart where, $10 \mathrm{~cm}$. plant to plant spacing was maintained. The eight pure lines of fenugreek were provided by the Acharya Narendra Deva University of Agriculture and Technology, Narendra Nagar (Kumarganj), Ayodhya (U.P.) India and two others commercial varieties collected from IARI, New Delhi and HAU, Hissar taken for this investigation. The observations were recorded on eleven characters viz, Days to $50 \%$ flowering, Days to maturity, Plant height $(\mathrm{cm})$, No of branches per plant, No. of pods per plant, Pod length $(\mathrm{cm})$, No. of seeds per pod, Biological yield per plant (g), Harvestindex (\%), 1000-seed weight (g), Seed yield per plant (g). The experimental data was compiled by taking the mean value of the 10 parents and $45 \mathrm{~F}_{1}$ 's and one standard check for all the 11 characters from all the three replications. Then it was subjected to the following statistical analyses. Phenotypic and genotypic correlation coefficients were worked out to study the relationship of various pairs of characters as suggested by Al-Jibouri et al. (1958) ${ }^{[1]}$.

\section{Result and Discussion}

Estimates of phenotypic and genotypic coefficient of correlation and that of direct and indirect effects provide the base, necessary for identification of traits of an ideal plant type and for indirect selection. The results on character association are presented in table 1, 1.1, 1.2, 2, 2.1 and 2.2.

At phenotypic level (table 1.2) seed yield per plant exhibited positively significant correlation with harvest index (0.821), Number of pods per plant (0.325), Number of branches per plant (0.278), plant height (0.337) and days to maturity (0.352). Harvest index exhibited positively significant correlation with test weight (0.481), plant height (0.412), days to maturity (0.321) and harvest index was also showed negatively significant correlation with biological yield per plant (-0.620). Biological yield per plant exhibited positively significant correlation with number of branches per plant (0.401), number of seed per pod (0.342) and showed negatively significant correlation with test weight $(-0.500)$ and plant height $(-0.269)$. Test weight showed negatively significant correlation with days to maturity (0.407) and days to $50 \%$ flowering $(0.630)$. Number of seeds per pod was exhibited positively significant correlation with number of pods per plant (0.648). Number of pods per plant exhibited positively significant correlation with number of branches per plant (0.297), plant height (0.291) and days to maturity (0.286). Days to maturity exhibited positively significant correlation with days to $50 \%$ flowering (0.570).

However, pod length, number of branches per plant and days to maturity showed non-significant correlation (positive or negative) with any of the characters during $E_{1}$.

At phenotypic level (table 1.2) seed yield per plant exhibited positively significant correlation with harvest index (0.828), Number of pods per plant (0.922), days to maturity (0.393), Number of branches per plant $(0.278)$, plant height $(0.335)$ and pod length (0.267). Harvest index exhibited positively significant correlation with test weight ( 0.483$)$, number of pod per plant (0.753), plant height (0.409) and days to maturity (0.356). Harvest index was also showed negatively significant correlation with biological yield per plant $(-0.614)$. Biological yield per plant exhibited positively significant correlation with number of branches per plant (0.403), number of seed per pod (0.340) and showed negatively significant correlation with test weight $(-0.500)$ and plant height $(-0.271)$. Test weight showed negatively significant correlation with days to $50 \%$ flowering (0.630) and days to maturity (0.405). Number of seeds per pod was exhibited positively significant correlation with number of pods per plant (0.633). Number of pods per plant exhibited positively significant correlation with number of branches per plant (0.294), plant height (0.287) and days to maturity (0.321). Days to maturity exhibited positively significant correlation with days to $50 \%$ flowering (0.548) during $\mathrm{E}_{2}$. 
Table 1: Estimates of phenotypic correlation coefficients among different characters in fenugreek (E1)

\begin{tabular}{|c|c|c|c|c|c|c|c|c|c|c|c|c|}
\hline $\begin{array}{l}\text { S. } \\
\text { No }\end{array}$ & Characters & \begin{tabular}{|c|} 
Days to \\
$\mathbf{5 0 \%}$ \\
Flowering \\
\end{tabular} & $\begin{array}{c}\text { Days to } \\
\text { Maturity }\end{array}$ & $\begin{array}{c}\text { Plant } \\
\text { Height } \\
\text { (cm) }\end{array}$ & \begin{tabular}{|c|}
$\begin{array}{c}\text { Number of } \\
\text { Branches/ } \\
\text { Plant }\end{array}$ \\
\end{tabular} & $\begin{array}{c}\text { Pod } \\
\text { length } \\
(\mathrm{cm}) \\
\end{array}$ & $\begin{array}{c}\text { Pods } \\
\text { /Plant }\end{array}$ & $\begin{array}{c}\text { Seeds/ } \\
\text { Pod }\end{array}$ & $\begin{array}{c}1000 \\
\text { Grain } \\
\text { Weight }\end{array}$ & $\begin{array}{c}\text { Biological } \\
\text { Yield/ } \\
\text { Plant (g) }\end{array}$ & $\begin{array}{c}\text { Harvest } \\
\text { Index } \\
(\%)\end{array}$ & \begin{tabular}{c|} 
Seed \\
Yield/ \\
Plant \\
\end{tabular} \\
\hline 1 & Days to $50 \%$ Flowering & 1 & $0.570 * *$ & 0.012 & -0.053 & 0.163 & -0.074 & -0.005 & $0.630 * *$ & -0.142 & 0.099 & 0.004 \\
\hline 2 & Days to Maturity & & 1 & 0.056 & -0.042 & 0.144 & $0.286^{*}$ & -0.187 & $0.407 * *$ & -0.082 & $0.321 *$ & $0.352 * *$ \\
\hline 3 & Plant Height $(\mathrm{cm})$ & & & 1 & 0.201 & 0.142 & $0.291 *$ & -0.108 & 0.252 & $-0.269^{*}$ & $0.412 * *$ & $0.337 *$ \\
\hline 4 & Branches/ Plant & & & & 1 & -0.091 & $0.297 *$ & 0.188 & 0.004 & $0.401 * *$ & -0.005 & $0.278 *$ \\
\hline 5 & Pods/ Plant & & & & & 1 & 0.217 & $0.648 * *$ & 0.062 & 0.095 & 0.195 & $0.325^{*}$ \\
\hline 6 & Pods Length $(\mathrm{cm})$ & & & & & & 1 & 0.029 & 0.157 & -0.052 & 0.746 & 0.921 \\
\hline 7 & Seeds/ Pod & & & & & & & 1 & -0.066 & $0.342 *$ & -0.095 & 0.11 \\
\hline 8 & 1000 Grain Weight & & & & & & & & 1 & $-0.500 * *$ & $0.481 * *$ & 0.246 \\
\hline 9 & Biological Yield/ Plant (g) & & & & & & & & & 1 & $-0.620 * *$ & -0.07 \\
\hline 10 & harvest Index (\%) & & & & & & & & & & 1 & $0.821 * *$ \\
\hline
\end{tabular}

Residual effect $=0.9893, * * *$ Significant at $5 \%$ and $1 \%$ probability level respectively

Table 1.1: Estimates of phenotypic correlation coefficients among different characters in fenugreek (E2)

\begin{tabular}{|c|c|c|c|c|c|c|c|c|c|c|c|c|}
\hline $\begin{array}{l}\text { S. } \\
\text { No }\end{array}$ & Characters & \begin{tabular}{|c|} 
Days to \\
$50 \%$ \\
Flowering
\end{tabular} & $\begin{array}{c}\text { Days to } \\
\text { Maturity }\end{array}$ & $\begin{array}{c}\text { Plant } \\
\text { Height } \\
(\mathrm{cm})\end{array}$ & \begin{tabular}{|c|}
$\begin{array}{c}\text { Number of } \\
\text { Branches/ } \\
\text { Plant }\end{array}$ \\
\end{tabular} & $\begin{array}{c}\text { Pod } \\
\text { length } \\
(\mathrm{cm})\end{array}$ & $\begin{array}{c}\text { Pods } \\
\text { /Plant }\end{array}$ & $\begin{array}{l}\text { Seeds/ } \\
\text { Pod }\end{array}$ & $\begin{array}{c}1000 \\
\text { Grain } \\
\text { Weight }\end{array}$ & \begin{tabular}{|c|} 
Biological \\
Yield/ \\
Plant (g)
\end{tabular} & $\begin{array}{c}\text { Harvest } \\
\text { Index } \\
(\%)\end{array}$ & \begin{tabular}{|c|} 
Seed \\
Yield/ \\
Plant \\
\end{tabular} \\
\hline 1 & Days to $50 \%$ Flowering & 1 & $0.548 * *$ & 0.013 & -0.055 & 0.159 & -0.074 & -0.004 & $0.630 * *$ & -0.142 & 0.09 & 0.004 \\
\hline 2 & Days to Maturity & & 1 & 0.074 & -0.03 & 0.143 & $0.321 *$ & -0.157 & $0.405 * *$ & -0.073 & $0.356^{* *}$ & $0.393 * *$ \\
\hline 3 & Plant Height $(\mathrm{cm})$ & & & 1 & 0.188 & 0.096 & $0.287 *$ & -0.113 & 0.251 & $-0.271^{*}$ & $0.409 * *$ & $0.335^{*}$ \\
\hline 4 & Branches/ Plant & & & & 1 & -0.122 & $0.294 *$ & 0.187 & 0.005 & $0.403 * *$ & -0.006 & $0.278^{*}$ \\
\hline 5 & Pods/ Plant & & & & & 1 & 0.153 & $0.633 * *$ & 0.047 & 0.058 & 0.178 & $0.267 *$ \\
\hline 6 & Pods Length $(\mathrm{cm})$ & & & & & & 1 & 0.031 & 0.157 & -0.049 & $0.753 * *$ & $0.922 * *$ \\
\hline 7 & Seeds/ Pod & & & & & & & 1 & -0.064 & $0.340 *$ & -0.084 & 0.113 \\
\hline 8 & 1000 Grain Weight & & & & & & & & 1 & $-0.500 * *$ & $0.483 * *$ & 0.246 \\
\hline 9 & Biological Yield/ Plant (g) & & & & & & & & & 1 & $-0.614 * *$ & -0.07 \\
\hline 10 & harvest Index (\%) & & & & & & & & & & 1 & $0.828 * *$ \\
\hline
\end{tabular}

Residual effect $=0.9948,{ }^{*}, * *$ Significant at $5 \%$ and $1 \%$ probability level respectively

At phenotypic level (table 2.2) seed yield per plant exhibited positively significant correlation with harvest index $(0.825)$, Number of pods per plant (0.921), days to maturity (0.374), Number of branches per plant (0.281), plant height (0.338) and pod length (0.287). Harvest index exhibited positively significant correlation with test weight $(0.483)$, number of pod per plant (0.750), plant height $(0.412)$ and days to maturity (0.339). Harvest index was also showed negatively significant correlation with biological yield per plant (-0.618). Biological yield per plant exhibited positively significant correlation with number of branches per plant (0.403), number of seed per pod (0.341) and showed negatively significant correlation with test weight $(-0.501)$ and plant height (-0.271). Test weight showed negatively significant correlation with days to $50 \%$ flowering (0.629) and days to maturity (0.406). Number of seeds per pod (0.698).Number of pod per plant was exhibited positively significant correlation with number of branches per plant (0.295), plant height (0.289) and days to maturity (0.304). exhibited positively significant correlation with days to $50 \%$ flowering (0.560).

However, days to $50 \%$ flowering was showed non-significant correlation (positive or negative) with plant height, number of branches per plant, pod length per plant, number of pods per plant, number of seeds per pod, biological yield per plant, harvest index and seed yield per plant. Days to maturity was showed non-significant correlation (positive or negative) with plant height, number of branches per plant, pod length, number of seed per pod and biological yield. Plant height was showed non-significant correlation (positive or negative) with number of branches per plant, pod length, number of seeds per pod and test weight. Number of branches per plant was showed non-significant correlation (positive or negative) with pod length per plant, number of seeds per pod, test weight and harvest index. Pod length was showed non-significant correlation (positive or negative) with number of pods per plant, test weight, biological yield and harvest index. Number of pods per plant was showed non-significant correlation (positive or negative) with seeds per pod, test weight and biological yield. Number of seeds per pod was showed nonsignificant correlation (positive or negative) with test weight, harvest index and seed yield per plant. Test weight was showed non-significant correlation (positive or negative) with seed yield per plant. Biological yield was showed nonsignificant correlation (positive or negative) with seed yield per plant during pooled.

Table 1.2: Estimates of Phenotypic correlation coefficients among different characters in fenugreek (pooled)

\begin{tabular}{|c|c|c|c|c|c|c|c|c|c|c|c|c|}
\hline $\begin{array}{l}\text { S. } \\
\text { No }\end{array}$ & Characters & \begin{tabular}{|c|} 
Days to \\
$\mathbf{5 0 \%}$ \\
Flowering \\
\end{tabular} & $\begin{array}{c}\text { Days to } \\
\text { Maturity }\end{array}$ & $\begin{array}{c}\text { Plant } \\
\text { Height } \\
\text { (cm) }\end{array}$ & $\begin{array}{c}\text { Number of } \\
\text { Branches/ } \\
\text { Plant } \\
\end{array}$ & $\begin{array}{c}\text { Pod } \\
\text { length } \\
(\mathrm{cm})\end{array}$ & $\begin{array}{l}\text { Pods } \\
\text { /Plant }\end{array}$ & $\begin{array}{c}\text { Seeds/ } \\
\text { Pod }\end{array}$ & $\begin{array}{c}1000 \\
\text { Grain } \\
\text { Weight }\end{array}$ & $\begin{array}{c}\text { Biological } \\
\text { Yield/ } \\
\text { Plant (g) }\end{array}$ & $\begin{array}{c}\text { Harvest } \\
\text { Index } \\
(\%)\end{array}$ & $\begin{array}{l}\text { Seed } \\
\text { Yield/ } \\
\text { Plant } \\
\end{array}$ \\
\hline 1 & Days to $50 \%$ Flowering & 1 & $0.560 * *$ & 0.012 & -0.055 & 0.122 & -0.074 & -0.005 & $0.629 * *$ & -0.142 & 0.095 & 0.004 \\
\hline 2 & Days to Maturity & & 1 & 0.066 & -0.037 & 0.051 & $0.304 *$ & -0.173 & $0.406^{* *}$ & -0.078 & $0.339 *$ & $0.374 * *$ \\
\hline 3 & Plant Height $(\mathrm{cm})$ & & & 1 & 0.19 & 0.105 & $0.289 *$ & -0.111 & 0.253 & $-0.271^{*}$ & $0.412 * *$ & $0.338^{*}$ \\
\hline 4 & Branches/ Plant & & & & 1 & -0.087 & $0.295 *$ & 0.188 & 0.005 & $0.403 * *$ & -0.006 & $0.281^{*}$ \\
\hline 5 & Pods/ Plant & & & & & 1 & 0.176 & $0.698 * *$ & -0.009 & 0.102 & 0.157 & $0.287 *$ \\
\hline 6 & Pods Length $(\mathrm{cm})$ & & & & & & 1 & 0.03 & 0.158 & -0.051 & $0.750 * *$ & $0.921 * *$ \\
\hline
\end{tabular}




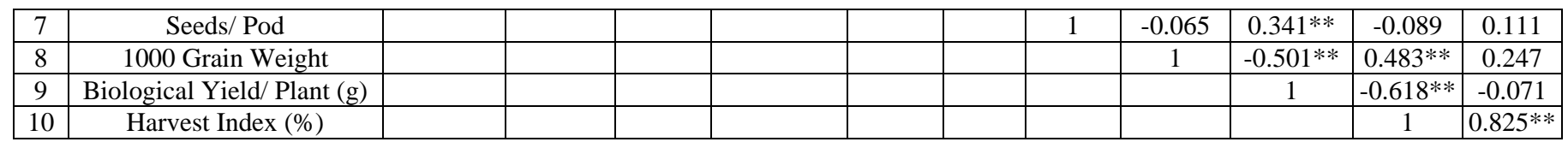

Residual effect $=0.9940, * * *$ Significant at $5 \%$ and $1 \%$ probability level respectively.

Table 2: Estimates of genotypic correlation coefficients among different characters in fenugreek (E1)

\begin{tabular}{|c|c|c|c|c|c|c|c|c|c|c|c|c|}
\hline $\begin{array}{l}\text { S. } \\
\text { No }\end{array}$ & Characters & \begin{tabular}{|c|} 
Days to \\
$\mathbf{5 0 \%}$ \\
Flowering
\end{tabular} & $\begin{array}{l}\text { Days to } \\
\text { Maturity }\end{array}$ & \begin{tabular}{|c|} 
Plant \\
Height \\
(cm)
\end{tabular} & $\begin{array}{c}\text { Number of } \\
\text { Branches/ } \\
\text { Plant }\end{array}$ & $\begin{array}{l}\text { Pod } \\
\text { length } \\
(\mathrm{cm})\end{array}$ & $\begin{array}{l}\text { Pods } \\
\text { /Plant }\end{array}$ & $\begin{array}{l}\text { Seeds/ } \\
\text { Pod }\end{array}$ & \begin{tabular}{|c|}
1000 \\
Grain \\
Weight
\end{tabular} & \begin{tabular}{|c|} 
Biological \\
Yield/ \\
Plant (g)
\end{tabular} & $\begin{array}{c}\text { Harvest } \\
\text { Index } \\
(\%)\end{array}$ & $\begin{array}{l}\text { Seed } \\
\text { Yield/ } \\
\text { Plant }\end{array}$ \\
\hline 1 & Days to $50 \%$ Flowering & 1 & 1.165 & 0.016 & -0.062 & 0.184 & -0.084 & -0.028 & 1.234 & -0.158 & 0.106 & 0.007 \\
\hline 2 & Days to Maturity & & 1 & 0.041 & -0.188 & $0.319^{*}$ & $0.549 * *$ & $-0.422 * *$ & $0.912 * *$ & -0.173 & $0.612 * *$ & $0.794 * *$ \\
\hline 3 & Plant Height $(\mathrm{cm})$ & & & 1 & 0.241 & 0.173 & $0.313^{*}$ & -0.127 & $0.445^{* *}$ & $-0.310^{*}$ & $0.451 * *$ & $0.366^{* *}$ \\
\hline \begin{tabular}{|l|}
4 \\
\end{tabular} & Branches/ Plant & & & & 1 & -0.136 & $0.351 * *$ & 0.256 & 0.045 & $0.524 * *$ & \begin{tabular}{|l|}
-0.003 \\
\end{tabular} & $0.339^{*}$ \\
\hline 5 & Pods/ Plant & & & & & 1 & 0.235 & $0.829 * *$ & 0.063 & 0.151 & 0.243 & $0.348 * *$ \\
\hline 6 & Pods Length $(\mathrm{cm})$ & & & & & & 1 & 0.027 & $0.297 *$ & -0.072 & $0.802 * *$ & 1.017 \\
\hline \begin{tabular}{|l|}
7 \\
\end{tabular} & Seeds/ Pod & & & & & & & 1 & -0.033 & $0.466 * *$ & -0.108 & 0.133 \\
\hline $8 \mid$ & 1000 Grain Weight & & & & & & & & 1 & -1.119 & $0.947 * *$ & $0.495 * *$ \\
\hline \begin{tabular}{|l|}
9 \\
\end{tabular} & Biological Yield/ Plant (g) & & & & & & & & & 1 & $-0.789 * *$ & -0.097 \\
\hline 10 & Harvest Index (\%) & & & & & & & & & & 1 & $0.953 * *$ \\
\hline
\end{tabular}

Residual effect $=0.8744, * * *$ Significant at $5 \%$ and $1 \%$ probability level respectively

Table 2.1: Estimates of genotypic correlation coefficients among different characters in fenugreek (E2)

\begin{tabular}{|c|c|c|c|c|c|c|c|c|c|c|c|c|}
\hline S. No & Characters & \begin{tabular}{|c|} 
Days to \\
$50 \%$ \\
Flowering
\end{tabular} & $\begin{array}{c}\text { Days to } \\
\text { Maturity }\end{array}$ & $\begin{array}{c}\text { Plant } \\
\text { Height } \\
(\mathrm{cm}) \\
\end{array}$ & $\begin{array}{c}\text { Branches/ } \\
\text { Plant }\end{array}$ & $\begin{array}{l}\text { Pods/ } \\
\text { Plant }\end{array}$ & \begin{tabular}{|c|} 
Pods \\
Length \\
$(\mathrm{cm})$
\end{tabular} & $\begin{array}{l}\text { Seeds/ } \\
\text { Pod }\end{array}$ & $\begin{array}{c}1000 \\
\text { Grain } \\
\text { Weight }\end{array}$ & $\begin{array}{c}\text { Biological } \\
\text { Yield/ } \\
\text { Plant (g) }\end{array}$ & \begin{tabular}{|c|} 
Harvest \\
Index \\
$(\%)$ \\
\end{tabular} & $\begin{array}{c}\text { Seed } \\
\text { Yield/ } \\
\text { Plant } \\
\end{array}$ \\
\hline 1 & Days to $50 \%$ Flowering & 1 & $0.932 * *$ & 0.025 & -0.065 & 0.178 & -0.088 & -0.026 & 1.155 & -0.194 & 0.095 & 0.009 \\
\hline 2 & Days to Maturity & & 1 & 0.109 & -0.149 & 0.22 & $0.518 * *$ & $-0.344 * *$ & $0.857 * *$ & $-0.431 * *$ & $0.578 * *$ & $0.723 * *$ \\
\hline 3 & Plant Height $(\mathrm{cm})$ & & & 1 & 0.215 & 0.118 & $0.313^{*}$ & -0.13 & $0.483 * *$ & $-0.479 * *$ & $0.443 * *$ & $0.433^{* *}$ \\
\hline 4 & Branches/ Plant & & & & 1 & -0.14 & $0.341 *$ & 0.239 & 0.046 & $0.711 * *$ & -0.015 & $0.351^{* *}$ \\
\hline 5 & Pods/ Plant & & & & & 1 & 0.174 & $0.818 * *$ & 0.079 & 0.051 & 0.211 & $0.359^{* *}$ \\
\hline 6 & Pods Length (cm) & & & & & & 1 & 0.032 & $0.280^{*}$ & -0.046 & $0.813 * *$ & 1.156 \\
\hline 7 & Seeds/ Pod & & & & & & & 1 & -0.035 & $0.813 * *$ & -0.078 & 0.247 \\
\hline 8 & 1000 Grain Weight & & & & & & & & 1 & -1.45 & $0.822 * *$ & $0.456^{* *}$ \\
\hline 9 & Biological Yield/ Plant (g) & & & & & & & & & 1 & -1.257 & $-0.391 * *$ \\
\hline 10 & Harvest Index (\%) & & & & & & & & & & 1 & $0.812^{* *}$ \\
\hline
\end{tabular}

Residual effect $=1.350, *$,* Significant at $5 \%$ and $1 \%$ probability level respectively

Table 2.2: Estimates of genotypic correlation coefficients among different characters in fenugreek (pooled)

\begin{tabular}{|c|c|c|c|c|c|c|c|c|c|c|c|c|}
\hline $\begin{array}{l}\text { S. } \\
\text { No }\end{array}$ & Characters & \begin{tabular}{|c|} 
Days to \\
$\mathbf{5 0 \%}$ \\
Flowering \\
\end{tabular} & $\begin{array}{c}\text { Days to } \\
\text { Maturity }\end{array}$ & \begin{tabular}{|c|}
$\begin{array}{c}\text { Plant } \\
\text { Height } \\
(\mathbf{c m})\end{array}$ \\
\end{tabular} & $\begin{array}{c}\text { Branches/ } \\
\text { Plant }\end{array}$ & $\begin{array}{c}\text { Pods } \\
\text { Length } \\
(\mathrm{cm})\end{array}$ & $\begin{array}{l}\text { Pods/ } \\
\text { plant }\end{array}$ & $\begin{array}{c}\text { Seeds/ } \\
\text { Pod }\end{array}$ & $\begin{array}{c}1000 \\
\text { Grain } \\
\text { Weight }\end{array}$ & \begin{tabular}{|c|} 
Biological \\
Yield/ \\
Plant (g)
\end{tabular} & \begin{tabular}{|c|} 
Harvest \\
Index \\
$(\%)$
\end{tabular} & $\begin{array}{l}\text { Seed } \\
\text { Yield/ } \\
\text { Plant }\end{array}$ \\
\hline 1 & Days to $50 \%$ Flowering & 1 & 1.014 & 0.024 & -0.065 & 0.151 & -0.086 & -0.028 & 1.182 & -0.148 & 0.1 & 0.007 \\
\hline 2 & Days to Maturity & & 1 & 0.078 & -0.165 & 0.021 & $0.518 * *$ & $-0.372 * *$ & $0.870 * *$ & -0.26 & $0.566 * *$ & $0.658^{* *}$ \\
\hline 3 & Plant Height $(\mathrm{cm})$ & & & 1 & 0.221 & 0.113 & $0.312 *$ & -0.153 & $0.466 * *$ & $-0.307 *$ & $0.446 * *$ & $0.375^{* *}$ \\
\hline 4 & Branches/ Plant & & & & 1 & -0.093 & $0.344 * *$ & 0.245 & 0.043 & $0.515 * *$ & -0.01 & $0.313^{*}$ \\
\hline 5 & Pods/ Plant & & & & & 1 & 0.186 & $0.894 * *$ & 0.017 & 0.145 & 0.186 & $0.323 *$ \\
\hline 6 & Pods Length $(\mathrm{cm})$ & & & & & & 1 & 0.03 & 0.288 & -0.042 & $0.798 * *$ & $0.992 * *$ \\
\hline 7 & Seeds/ Pod & & & & & & & 1 & -0.033 & $0.534 * *$ & -0.091 & 0.173 \\
\hline 8 & 1000 Grain Weight & & & & & & & & 1 & -1.061 & $0.869 * *$ & $0.432 * *$ \\
\hline 9 & Biological Yield/ Plant (g) & & & & & & & & & 1 & $-0.807 * *$ & -0.106 \\
\hline 10 & Harvest Index $(\%)$ & & & & & & & & & & 1 & $0.887 * *$ \\
\hline
\end{tabular}

Residual effect $=0.9480, * * *$ Significant at $5 \%$ and $1 \%$ probability level respectively

Thus it can be inferred that selection based on any one of these traits either alone or in combination will result in identifying high yielding strains. However, association among other traits were observed in present study were, days to $50 \%$ flowering showed significant and positive association with days to maturity; highly significant and positive correlation among biological yield per plant and pod length; plant height was strongly associated with seeds per pod and harvest index was significantly correlated with seed yield per plant, these association might be due to linkage of genes determining these traits. These results are in general agreement with Singh and Kaur (2007) [ ${ }^{12]}$, Dashora et al. (2011) ${ }^{[4]}$, Pushpa et al. (2012) ${ }^{[10]}$, Yadav et al. (2013) ${ }^{[14]}$ and Patahk et al. (2014) ${ }^{[8]}$.
Positive correlation of seed yield per plant with harvest Index suggested that yield could be improved substantially through improving the high degree of harvest Index. These results are in conformity with findings of Prajapati et al. (2010), Pushpa et al. (2012) ${ }^{[10]}$ and Yadav et al. (2013) ${ }^{[14]}$.

Improvement in these traits could definitely improve the productivity as they showed positive association with grain yield. Based on the estimates of genotypic and phenotypic correlation, the breeder would also be able to decide the method of breeding to be followed so that the useful correlation could be exploited and the undesirable ones may be modified by generating fresh variability to obtain new recombination. 
Thus, from the present study on correlation, it was apparent that for improvement in fenugreek improvement in six traits viz; plant height, pods per plant, seeds per pod, number of branches per plant, harvest Index, and 1000-grain weight are more important since, they have highest correlation coefficient values with yield during both seasons $\left(E_{1}, E_{2}\right)$ and pooled.

\section{Reference}

1. Al-Jibouri HA, Miller, Robinson HF. Agron. J. 1958; 50:633-636.

2. Allard RW. Principles of Plant Breeding. John Wiley and Sons Inc., New York, 1960.

3. Aykroyd WR. The nutritive value of Indian foods and planning for statisfactory diets. ICMR Special Report, Series No. 42, 1963.

4. Dashora A, Maloo SR, Dashora LK. Variability, correlation and path coefficient analysis in fenugreek (Trigonella foenum-graecum L.) under water limited condition. Journal of Spices and Aromatic Crops. 2011; 20(1):38-42.

5. Gill SS, Singh H. Effect of planting date and leaf cutting on the seed yield of methi (Trigonella foenum-graecum L.). J Res. Punjab Agric., Univ., Ludhiana. 1988; 25:206209.

6. Jatasra DS, Lodhi GP. Suitable fodder for grain crop for late sowing. Haryana Fmg. 1980; 9(12):7.

7. Pandey SC. Improvement of leafy vegetables, In: Advances in Horticulture (K.L. Chadha and G. Kallo, Eds) Malhotra Publishing House. New Delhi, 1993, 325342.

8. Patahk AR, Patel AI, Joshi HK, Patel DA. Genetic variability, correlation and path coefficient analysis in fenugreek (Trigonella foenum-graecum L.). Trends in Biosciences. 2014; 7(4):234-237.

9. Prajapati DB, Ravindrababu Y, Prajapati BH. Genetic variability and character association in fenugreek (Trigonella foenum-graecum L.). Journal of Spices and Aromatic Crops. 2010; 19(1/2):61-64.

10. Pushpa TN, Chandregowda M, Srikantaprasad D, Gowda APM. Evaluation of fenugreek (Trigonella foenumgraecum L.) genotypes for growth and seed yield. Crop Research (Hisar). 2012; 43(1/2/3):238-244.

11. Rao PU, Sharma RD. An evaluation of protein quality of fenugreek seed (Trigonella foenum-graecum L.) and their supplementary effects. Food Chemistry. 1987; 24(1):1-9.

12. Singh P, Kaur A. Genetic evaluation of Trigonella foenum-graecum $\mathrm{L}$. for seed yield and quality attributes. Crop Improvement. 2007; 34(1):90-94.

13. Som MG, Maity TK. Fenugreek In: Vegetable crops in India (T.K. Bose and M.G. Som, eds.) Naya Prakash. Calcutta, India, 1986, 680-686.

14. Yadav Yogendra, Yadava PS, Pandey VP, Adesh Kumar. Genetic variability, correlation and path co-efficient analysis studies in fenugreek (Trigonella foenumgraecum L.). Asian Journal of Horticulture. 2013; $8(2): 456-459$. 